\title{
What should not be in a manuscript submitted to a scientific journal
}

\author{
Martin Caon ${ }^{1}$
}

Published online: 30 May 2015

(C) Australasian College of Physical Scientists and Engineers in Medicine 2015

So many authors have written about how to write a scientific article or prepare a manuscript for publication that I will not repeat their work or attempt to cite them here. On the other hand, relatively few have written about how not to write, see for example Oded [1]. And Sand-Jensen has humorously written about (I think) how not to not write an article for publication [2]—but it is difficult to know for certain;-). Thanks to the benefits of peer review and to the writing ability of the authors, the published literature has many examples of good writing. However, unless you are a journal editor or do not get a lot or manuscripts to review, you may not see examples of poorly prepared manuscripts. Drawing from the examples that have crossed my screen, I will suggest some things that should not be in a manuscript when submitting it to a scientific journal.

Do not think that an appropriate literature review is merely a list of authors with the title of their articles paraphrased. For example the following (with alterations to preserve anonymity):

Van $* * * * *$ et al. [6] noted the potential effect of XY size and shape on the $\mathrm{ABC}$ distribution. $\mathrm{N}^{* * * * *}$ et al. [7] examined the effect of various thicknesses of $X Y$ coupling layers on the ABC patterns from dual concentric conductor based on conformal microwave array superficial hyperthermia applicators. De $* * * * * * *$ et al. [8] presented the effects of XY dimension and configuration on the effective field size of the Lucite cone applicator for superficial hyperthermia. $* * * * * * * *$ ******* and $* * * * * *$ [9]

Martin Caon

martin.caon@flinders.edu.au

1 Flinders University, School of Health Sciences, Adelaide, Australia numerically computed and compared both the ABC distributions and effective field size in the presence and absence of XY. ***** et al. [10] investigated the influence of $\mathrm{XY}$ temperature on the thermal distribution inside a homogeneous muscles phantom for a microwave applicator for superficial hyperthermia on small subcutaneous tumors;

provides no clue about the significance of the articles to the work being reported. In your Introduction make the achievements of the article being cited, why you are citing it and how it relates to your present work quite clear.

Do not cite an excessive number of articles at the end of a sentence in your Introduction to support a minor point (I have seen eleven cited). This is the equivalent of throwing the bundle of articles into the air and after they have hit the ground, challenging the reader to find something of relevance amongst the resulting mess. It does not help the interested reader to find more information. Rather, demonstrate your understanding of the literature by selecting the one or two articles that illustrate your argument well, and direct the reader to them. If you need to cite another article, write another sentence.

Do not provide a literature review that refers only to work that was published ten (or more) years ago. That is, do not fail to keep abreast of the literature in your field. A manuscript that fails to cite recently published articles is unlikely to be reporting novel work. Perhaps there is a reason why nothing less than 10 years old is being citedand the reason is not the your neglect of the literature. If this is the case then explain why.

Do not "cut and paste" into your manuscript, anything from the published literature that you find on the internet. At all. Ever. Submitted manuscripts are routinely searched for copied text and such text is easy to detect. The practice 
is lazy, it means that your manuscript is not novel, it will ruin your reputation and, to emphasise the point by understatement, is viewed unfavourably by editors. Furthermore copying text is plagiarism and usually leads to your manuscript being rejected without being sent to reviewers.

Do not submit to a journal a manuscript containing material that has already been published as a four or five page conference paper. Such conference papers are publications and are easily located on the internet. So submitting the same work to a journal is considered an attempt at duplicate publication and to be misconduct.

Do not "slice the salami". Some authors submit manuscripts that are almost duplicates but differ in the reagent, or isotope or some other variable. Sometimes the results of an investigation will be divided into the "least publishable unit" and spread thinly across several manuscripts when one would suffice. The people whose opinion of your work matters, will be more impressed by one article with substance and quality than by several mediocre ones.

Do not use uncommon acronyms excessively, or even a lot. They are not easy to remember and very difficult to read. Here is an example from another journal: "SPE avoidance is assumed by FAA to occur with SWPE alerts, but SWPC missed $13 \%$ of the SPE that caused GLE, and the FAA does not require compliance with SWPC alerts." And an example from this journal: "For dMLC IMRT plans calculated with Dm option, it is found that for CTV and PTV, Dmaximum (Dmax) and Dmedian (Dmed) values..." Rather, construct the sentence so that repeating acronyms is not required and write comprehensible sentences rather than sentences full of jargon.

Do not use chatty, conversational or colloquial expressions when your intention is to be precise. Don't assume that your reader shares your understanding of such expressions. If your writing is ambiguous, the reader is left to guess at your meaning. While this is appropriate for poetry, it doesn't get the job done in a scientific article.

Do not state your number data to five or six decimal places unless that level of certainty is warranted. Do not use lists of dot points where a description written in prose is more enlightening. Do not include spelling errors.

Do not leave it to the reader to identify the major results from your data tables. State clearly the major results that form the basis of the work being reported. Do not fail to discuss in the Discussion section the results that are presented in the Results section. These results will be the ones that are interpreted, explained and compared with the published results of others in your Discussion. Do not combine the Results section with the Discussion section. Your discussion will convince the editor and reviewer that you have something of consequence to report, what its significance is and what applicability it has.

I offer the following advice to authors who are not native writers of English and who are attempting the difficult task of writing about their work in English. If you want your manuscript to be rejected, do not have your manuscript edited for English expression by a native speaker of English (who has an understanding of your field). When editing by a native English speaker is requested by the editor, do not fail to comply. A colleague more fluent in written English than the author(s), but who is not a native speaker of English will probably not do. Many reviewers have little patience for a manuscript that is difficult to follow because the English expression is inadequate. As good reviewers are a valuable commodity, an editor does not wish to antagonise them. Nor does an editor wish to tarnish the reputation of the journal by sending underprepared manuscripts to reviewers. Consequently, this editor will not accept a manuscript for review until the written English is acceptable.

\section{References}

1. Goldreich O (1996) How NOT to write a paper, www.cs.iastate. edu/ honavar/write-not.pdf

2. Sand-Jensen K (2007) How to write consistently boring scientific literature. Oikos 116:723-727. doi:10.1111/j.2007.0030-1299.156 74.x 